\title{
Case Report: Treatment Resistant Ipilimumab Related Colitis in an Elderly Metastatic Melanoma Patient
}

\author{
Nicolas Maréchal ${ }^{1 *}$, Sofie Wilgenhof ${ }^{2}$, Bart Neyns², Veerle Morlion ${ }^{3}$, Daniel Urbain ${ }^{4}$, Tony Mets ${ }^{1}$ and Ingo Beyer ${ }^{1}$ \\ ${ }^{1}$ Department of Geriatric Medicine, Universitair Ziekenhuis Brussel, Vrije Universiteit Brussel (VUB), Belgium \\ ${ }^{2}$ Department of Medical Oncology, Universitair Ziekenhuis Brussel, Vrije Universiteit Brussel (VUB), Belgium \\ ${ }^{3}$ Department of Pathology, Universitair Ziekenhuis Brussel, Vrije Universiteit Brussel (VUB), Belgium \\ ${ }^{4}$ Department of Gastroenterology, Universitair Ziekenhuis Brussel, Vrije Universiteit Brussel (VUB), Belgium
}

\begin{abstract}
Background: Ipilimumab is a fully humanized monoclonal antibody against cytotoxic T-lymphocyte-associated antigen 4 (CTLA-4). In 2010, ipilimumab became the first systemic therapy to improve the overall survival of patients with advanced melanoma in two randomized phase III clinical trials. Related to its immune-cell activating mode of action, ipilimumab therapy is associated with a spectrum of immune-related adverse events (irAEs), most commonly located at the skin and gastrointestinal tract. IrAEs are managed by withdrawal of ipilimumab and early administration of high-dose corticosteroids. Ipilimumab-related colitis, refractory to corticotherapy has been reported to respond readily to treatment with the anti-tumor-necrosis-factor monoclonal antibody infliximab.
\end{abstract}

Case report: We describe a case of severe ipilimumab-related colitis in an elderly patient with metastatic melanoma, responding neither to high-dose corticosteroids, nor to infliximab, and resolving only after the washout of ipilimumab. Several complications related to immunosuppression were encountered during this period including recurrent urinary tract infections, herpes simplex stomatitis, pneumonia, oropharyngeal candidiasis, and catheter septicaemia.

Conclusion: Now that immunotherapy for cancer becomes available also for the older patient, irAEs will be more frequently encountered by geriatricians. Although the treatment guidelines remain unchanged for older patients, our case demonstrates that older patients may not react easily to standard treatment. Early diagnosis of the irAEs and prompt withdrawal of ipilimumab are essential. Hence, geriatricians may have to play a crucial role for optimal management of these patients.

Keywords: Case report; Ipilimumab; Immune-related adverse events; Corticotherapy-resistant colitis; Anti-tumor-necrosis-factor resistant colitis

Abbreviations: CTLA-4: T-Lymphocyte-Associated Antigen-4; irAE: Immune-Related Adverse Events; FDG: Fluorodeoxyglucose; PET-CT: Positron Emission Tomography-Computed Tomography; TNF - $\alpha$ : Tumor Necrosis Factor-Alpha; PCR: Polymerase Chain Reaction; CMV: Cytomegalovirus

\section{Introduction}

Ipilimumab is a fully humanized monoclonal antibody against T-lymphocyte-associated antigen-4 (CTLA-4), an inhibitory receptor on cytotoxic T-cells, whose blockade augments anti-tumour immune responses, hence inducing tumour regression [1]. Ipilimumab has been extensively studied in phase II clinical trials and it has recently been shown to improve the overall survival of advanced melanoma patients in two randomized controlled phase III trials $[2,3]$. In previously treated melanoma patients, ipilimumab monotherapy $(3 \mathrm{mg} / \mathrm{kg})$ prolonged the median overall survival from 6.4 months (in patients treated with a gp100 peptide vaccine) to 10.1 months [2]. In previously untreated patients, ipilimumab $(10 \mathrm{mg} / \mathrm{kg})$ in combination with dacarbazine improved survival as compared to dacarbazine alone (11.2 vs 9.2 months) [3]. In 2011, ipilimumab was approved by the European Medicines Agency (EMA), for pretreated melanoma patients, at a dose of $3 \mathrm{mg} / \mathrm{kg}$ every three weeks for a total of four infusions.

Related to its immune-activating mode of action, various ipilimumab-associated immune mediated inflammatory disorders affecting normal organs and tissues have been observed. These have been commonly referred to as "immune-related adverse events" (irAEs). Most common irAEs are observed at the skin (dermatitis) and gastrointestinal tract (colitis). Less frequent are immune-related hepatitis, endocrinopathies (hypophysitis, thyroiditis), neurological
(Guillain Barré syndrome, sensory and motor neuropathy), and ocular (uveitis, iritis) irAEs [4]. IrAEs can occur at any point during ipilimumab treatment, colitis occurring most often following the third or fourth dose. Algorithms have been developed for managing most common irAEs $[4,5]$.

Any changes in bowel habits (diarrhea, blood or mucus in stool, abdominal pain, and even peritoneal signs due to bowel perforation) should be considered to be potential symptoms of gastrointestinal ipilimumab-related irAEs in patients treated with this agent. Management of severe (grade 3 or 4 ) colitis relies on prompt stopping of ipilimumab administration, treatment with high-dose corticosteroids, and supportive measures. Most patients will respond favorably within days to such measures. Prophylactic use of local acting corticosteroids (budenoside, entocort EC) failed to prevent the onset of gastrointestinal irAEs compared with placebo in patients treated with ipilimumab [6]. The median duration of treatment in grade 3-4 irAEs of the gastrointestinal tract is 2.3 weeks (ranging up to 13.9 weeks)

*Corresponding author: Nicolas Maréchal, Department of Geriatric Medicine Universitair Ziekenhuis Brussel, Vrije Universiteit Brussel (VUB), UZ Brussel, Laarbeeklaan 101, 1090 Brussels, Belgium, Tel: +32 247763 66; E-mail: nicolas.marechal@uzbrussel.be

Received February 13, 2018; Accepted February 27, 2018; Published February 28, 2018

Citation: Maréchal N, Wilgenhof S, Neyns B, Morlion V, Urbain D, et al. (2018) Case Report: Treatment Resistant Ipilimumab Related Colitis in an Elderly Metastatic Melanoma Patient. J Gerontol Geriatr Res 7: 461. doi:10.4172/21677182.1000461

Copyright: (c) 2018 Maréchal N, et al. This is an open-access article distributed under the terms of the Creative Commons Attribution License, which permits unrestricted use, distribution, and reproduction in any medium, provided the original author and source are credited. 
followed by corticosteroid taper [7]. Corticotherapy refractory cases have been reported to respond to treatment with one to two doses of intravenous infliximab, a chimeric IgG antibody against tumour necrosis factor- $\alpha[8]$.

Systemic corticosteroids do not affect the anti-tumoral activity achieved by CTLA-4 inhibition, but the impact of infliximab remains unknown.

Successful treatment of ipilimumab related colitis with corticosteroids and infliximab have been reported in the age group less than 65 years, but not yet in very elderly patients exhibiting treatmentrefractory colitis.

We here report a case of severe ipilimumab-related colitis (grade 3) in an elderly metastatic melanoma patient that did not respond to highdose corticosteroids, and one dose of infliximab. Protracted supportive therapy including treatment for a number of infectious complications was needed in order to obtain a slow recovery.

\section{Case Presentation}

An 82-year-old female patient, was initially (November 2011) diagnosed with a superficially spreading melanoma (Breslow thickness $2.6 \mathrm{~mm}$, Clark level IV) located at her right lateral ankle (Figure 1a). It was surgically treated in December 2011. An inguinal sentinel biopsy revealed metastasis, but subsequent inguinal-iliac lymph node dissection was negative, as was a $18 \mathrm{~F}$-fluorodeoxyglucose positron emission tomography/computed tomography (FDG-PET/CT).

The patient's medical history included medically controlled dyslipidemia, hypertension and glaucoma.

In October 2012 she presented with progressive unexcisable multiple subcentimetric in-transit metastases on her right thigh (Figure 1b). Two biopsies were performed of the cutaneous lesions and histologically confirmed the suspicion of subcutaneous metastases. No additional metastatic sites were seen on FDG-PET/CT. A wildtype BRAF status was found (no NRAS or c-kit mutation analysis was performed), and treatment with temozolomide was initiated, with stabilization of the metastatic lesions. Treatment was discontinued after four cycles due to grade 3 toxicity (nausea, vomiting, and anorexia) and the absence of tumour response. Subsequently, ipilimumab treatment ( $3 \mathrm{mg} / \mathrm{kg} / 3$ weeks) was started. Three weeks after the second ipilimumab administration, grade 2 diarrhoea developed. The third ipilimumab

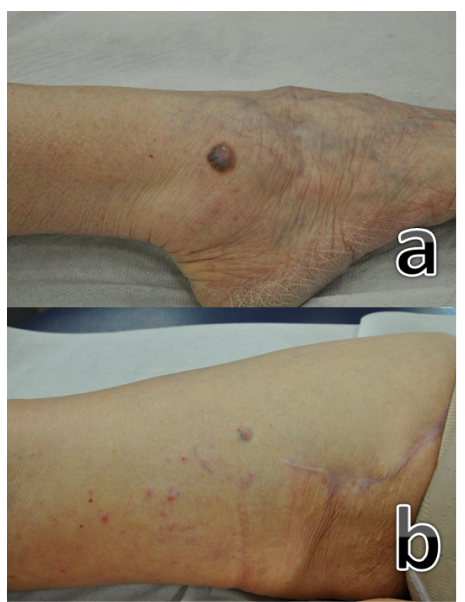

Figure 1: Superficial melanoma (a) right ankle (November 2011), and (b) right thigh (October 2012).

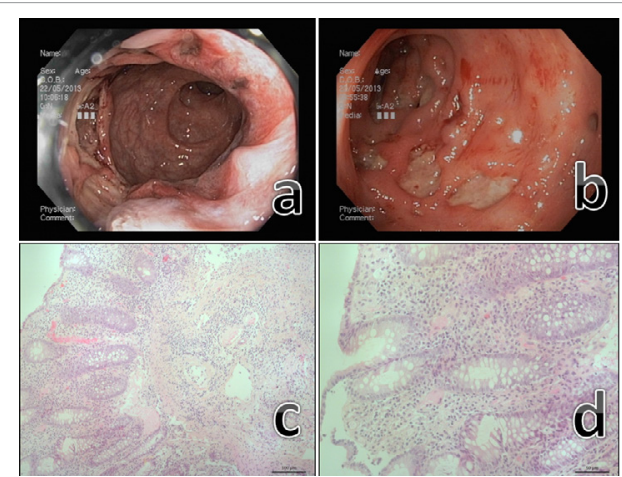

Figure 2: Colonoscopy showing deep (a) anal and (b) rectosigmoid necrotic ulcerations; (c, d) diffuse invasion of the lamina propria (rectosigmoid segment) with inflammatory cells (lymphocytes); focal ulceration and fibrine formation. Immunohistology was negative for CMV infection.

administration was withheld and $32 \mathrm{mg}$ oral methylprednisolone was initiated.

Four days later she had markedly improved and reported only one stool over 24 hours. Methylprednisolone was continued at the same dose. One week later, the patient presented with grade 3 diarrhea anorexia and weakness. She had stopped corticosteroid treatment for 3 days on her own initiative because symptoms had worsened.

Laboratory blood results showed strong elevation of C-reactive protein $(208.1 \mathrm{mg} / \mathrm{dL})$, with a normal white blood cell count. Stool cultures were normal, with negative results for Clostridium Difficile toxin (fecal leukocytes and Clostridium Difficile PCR analyses were not performed). Colonoscopy revealed anal and rectosigmoid ulcerations (Figures $2 \mathrm{a}$ and $2 \mathrm{~b}$ ). Biopsies of the (recto)sigmoid showed invasion of the lamina propria with inflammatory cells (lymphocytes), focal ulceration and fibrine formation, compatible with ipilimumab-induced colitis (Figures $2 \mathrm{c}$ and $2 \mathrm{~d}$ ). Immunohistology was not suggestive for cytomegalovirus (CMV) infection. No iatrogenic adrenal insufficiency was suspected.

According to established algorithms for this irAE, the patient was treated with high dose intravenous corticosteroids (methylprednisolone $40 \mathrm{mg}$ BID). For the following 6 days her symptoms did not improve and infliximab (Remicade, Centocor Ortho Biotech Inc, Malvern, PA; $5 \mathrm{mg}$ / $\mathrm{kg}$ ) was administered. Two days later she was no longer experiencing abdominal pain but diarrhea (grade 3 ) had not improved. Six days after the infliximab administration a colonoscopy showed no improvement, thus necessitating continuation of high-dose methylprednisolone and total parenteral nutrition. For ten days the patient was treated with intravenous corticosteroids (methylprednisolone $40 \mathrm{mg}$ BID). The following two weeks methylprednisolone was given orally $(16 \mathrm{mg}$ BID). However, after two weeks treatment with oral corticosteroids, an increase of frequency of diarrhea and abdominal pain was seen, again necessitating administration of methylprednisolone for another ten days intravenous (40 mg BID). The following five weeks there was a gradual resolution of the diarrhea. Methylprednisolone could be tapered slowly (12 mg per week) and could be completely stopped at discharge ( 9 weeks after admission).

Several hospital-acquired infections prevented the administration of a second dose of infliximab. Recurrent urinary tract infections (UTI) with Klebsiella pneumoniae complex and Proteus mirabilis were both treated with amoxicillin clavulanic acid, UTI with Pseudomonas aeruginosa was treated with ciprofloxacin. Furthermore the patient presented with herpes simplex stomatitis (treated with acyclovir for 
10 days (500 mg TID IV)), pneumonia (treated with piperacilline/ tazobactam for 10 days ( $4 \mathrm{~g}$ TID IV)), oropharyngeal candidiasis (treated with fluconazole for 10 days $100 \mathrm{mg}$ QD PO) and a central venous catheter septicemia (Staphylococcus epidermidis) (treated with vancomycine IV intermittent according to serum drug level).

Hyperglycemia necessitated insulin treatment. Five weeks after the administration of infliximab, colonoscopy showed a normal mucosa. The intransit metastases on the right leg had remained stable and a follow-up CT scan 12 week after the initiation of ipilimumab did not indicate new metastases. Two months after admission, the patient was discharged to a rehabilitation facility.

Total-body FDG-PET/CT (20 weeks after initiation ipilimumab) showed an increase of mediastinal lymph nodes. Ipilimumab treatment was not re-initiated due this severe irAE. No other treatment for the metastatic melanoma was started. The patient was re-admitted (November 2013) 4 months after discharge because of acute renal failure and respiratory distress. CT scan showed bilateral pleural effusion and further increase of mediastinal lymph nodes (suggesting progressive metastatic disease). In consultation with the patient palliative care was started. She died 9 days after admission.

\section{Discussion}

We describe a case of severe ipilimumab-related treatmentrefractory colitis in an elderly metastatic melanoma patient, followed by complications of the immunosuppressive treatment.

Colitis only resolved after two months of treatment with highdose systemic corticosteroids and a single infusion of infliximab. Grade 3 or 4 ipilimumab-associated irAEs of the gastro-intestinal tract can be observed in $8 \%-23 \%$ of patients, but early administration of corticosteroids generally leads to significant improvement of symptoms within one to two weeks [4].

For patients with refractory symptoms despite treatment with high dose corticosteroids for approximately five days, a single infusion of infliximab $5 \mathrm{mg} / \mathrm{kg}$ may be considered and can be repeated (every two weeks). In exceptional cases (persisting symptoms despite corticosteroids and infliximab) a diverting ileostomy or colectomy may be considered as a last resort $[8,9]$.

In our patient colitis persisted for several weeks and was resistant to high-dose corticosteroids and infliximab treatment. Infliximab infusion could not be safely repeated as it might have aggravated the immunosuppressed state of our patient, illustrated by several hospitalacquired bacterial, fungal, and viral infections. Complete resolution of diarrhea, and anal and rectosigmoid ulcerations was only observed after two months. Taking into account the half-life of ipilimumab (14 days), recovery was likely to occur only following complete elimination of ipilimumab in this patient. Therefore, corticosteroids had to be administrated during the whole period of active colitis in order to limit severity of symptoms, regardless of the infectious complications.

Initially, methylprednisolone was started for grade 2 diarrhea and a favorable response was seen. However, at recurrence of symptoms the patient did not notify her care-givers and had stopped corticotherapy three days prior to her admission. Hence, our case illustrates that adequate patient communication and education are of major importance in the management of ipilimumab-induced irAEs. Physicians should urge for immediate notification in case worsening of symptoms occurs following an initial response to corticotherapy [10].

The triggering event and immune-etiology of the ipilimumabrelated colitis remains unclear [11]. Ipilimumab seems to induce a unique pattern of dysregulation of the gastrointestinal mucosal immunity, distinct from those observed in classic inflammatory bowel disease or graft-versus-host disease [12].

The immune status in the aging population is very different compared to young individuals due to the immunoscenescence, compromised homeostasis, and higher anergic lymphocytic turnover. The pro-inflammatory immune environment with severe lymphopenia could lead to the more severe irAEs after the treatment with immunomodulatory and/or immunosuppressive agents.

Safety and efficacy of administration of ipilimumab in elderly patients ( $>65$ years) do not significantly differ in comparison with younger patients $[13,14]$. Toxicity rates in the use of immune checkpoint inhibitors are not higher in the older population. Moreover, in comparison to traditional chemotherapy, use of novel immunotherapies in the geriatric population offer a better side effect profile, with lower impact on quality of life and functional status $[15,16]$. Currently, there are only limited data from the literature available to guide physicians in treating patients with immune checkpoint inhibitors in advanced cancer and a low performance status. Additional prospective studies are needed $[14,15]$.

Our case illustrates that prolonged administration of high-dose corticosteroids for the management of ipilimumab-related irAEs, may lead to immunosuppression and put patients at risk for hospital acquired and opportunistic infections. Furthermore, additional complications (e.g. diabetes mellitus) can occur, especially in older and more fragile patients, as illustrated in our case. In order to avoid or limit these complications prophylactic treatments such as trimethoprim/ cotrimoxazole for Pneumocystis carinii pneumonia, or acyclovir for herpes simplex infections could be considered. In retrospect, in our patient this type of preventive treatment might at most have prevented one of the UTI episodes and the stomatitis, but not the other infections. Another possibility to avoid infectious complications would be to reduce duration and dose of steroids as much as possible, by using infliximab (and/or other TNF-alpha antagonists) as first line therapy. To our best knowledge this has not yet been recommended, even though age-specific aspects in the use of immune checkpoints inhibitors have recently been addressed, indicating an interesting topic for further research [14].

\section{Conclusion}

Immunotherapy for cancer and melanoma in particular, is a rapidly developing new field, showing great promise to become a new therapeutic pillar in oncology. Besides ipilimumab, other new immunotherapeutic agents that have the potential for eliciting irAEs are currently under development in clinical trials. As the population receiving immunotherapy for cancer grows older, geriatricians will be more commonly confronted with irAEs. Because early recognition and algorithm-based management are of major importance, all clinicians taking care of older adults should be aware of this new class of potentially life-threatening irAEs.

\section{Consent}

Consent to publish was obtained from the institutional review board (IRB Belgium O.G. 016) UZ Brussel.

\section{Acknowledgements}

No acknowledgements.

\section{References}

1. Leach DR, Krummel MF, Allison JP (1996) Enhancement of antitumor immunity by CTLA-4 blockade. Science 271: 1734-1736. 
Citation: Maréchal N, Wilgenhof S, Neyns B, Morlion V, Urbain D, et al. (2018) Case Report: Treatment Resistant Ipilimumab Related Colitis in an Elderly Metastatic Melanoma Patient. J Gerontol Geriatr Res 7: 461. doi:10.4172/2167-7182.1000461

2. Hodi F, O'Day S, McDermott D (2010) Improved survival with ipilimumab in patients with metastatic melanoma. N Engl J Med 363: 711-723.

3. Robert C, Thomas L, Bondarenko I (2011) Ipilimumab plus dacarbazine for previously untreated metastatic melanoma. N Engl J Med 364: 2517-2526.

4. Weber JS, Kahler KC, Hauschild A (2012) Management of immune-related adverse events and kinetics of response with ipilimumab. J Clin Oncol 30: 2691 2697.

5. Wilgenhof S, Pierret L, Corthals $\mathrm{J}$ (2011) Restoration of tumor equilibrium after immunotherapy for advanced melanoma: three illustrative cases. Melanoma Res 21: 152-159.

6. Weber J, Thompson JA, Hamid O (2009) A randomized, double-blind, placebocontrolled, phase II study comparing the tolerability and efficacy of ipilimumab administered with or without prophylactic budesonide in patients with unresectable stage III or IV melanoma. Clin Cancer Res 15: 5591-5598.

7. Fecher LA, Agarwala SS, Hodi FS (2013) Ipilimumab and its toxicities: a multidisciplinary approach. Oncologist 18: 733-743.

8. Pagès C, Gornet JM, Monsel G (2013) Ipilimumab-induced acute severe colitis treated by infliximab. Melanoma Res 23: 227-230.

9. Bostwick AD, Salama AK, Hanks BA (2015) Rapid complete response of metastatic melanoma in a patient undergoing ipilimumab immunotherapy in the setting of active ulcerative colitis. J Immuno Therapy Cancer 3: 19.

10. O'Day S, Weber JS, Wolchok JD (2011) Effectiveness of treatment guidance on diarrhea and colitis across ipilimumab studies. ASCO Meeting Abstracts 29: 8554.

11. Lord JD, Hackman RC, Moklebust A (2010) Refractory colitis following antiCTLA4 antibody therapy: analysis of mucosal FOXP3+ T cells. Dig Dis Sci 55: 1396-1405.

12. Berman D, Parker SM, Siegel J (2010) Blockade of cytotoxic T-lymphocyte antigen-4 by ipilimumab results in dysregulation of gastrointestinal immunity in patients with advanced melanoma. Cancer Immun 10: 11.

13. Yervoy (ipilimumab) Injection, prescribing information Princeton, N.J: BristolMyers Squibb. March 2011; Available at: http://packageinserts.bms.com. Accessed January 18, 2012.

14. Johnson DB, Sullivan RJ, Menzis AM (2017) Immune checkpoints inhibitors in challenging populations: Cancer.

15. Elias R, Morales J, Rehmans Y (2016) Immune Checkpoint Inhibitors in Older Adults. Curr Oncol Rep 18-47.

16. Pawelec G (2017) Immunosenescence and Cancer. Biogerontology. 